\title{
Distance-regular graphs with complete multipartite $\mu$-graphs and AT4 family
}

\author{
Aleksandar Jurišić · Jack Koolen
}

Received: 28 December 2005 / Accepted: 30 October 2006 /

Published online: 10 January 2007

(C) Springer Science + Business Media, LLC 2007

\begin{abstract}
Let $\Gamma$ be an antipodal distance-regular graph of diameter 4, with eigenvalues $\theta_{0}>\theta_{1}>\theta_{2}>\theta_{3}>\theta_{4}$. Then its Krein parameter $q_{11}^{4}$ vanishes precisely when $\Gamma$ is tight in the sense of Jurišić, Koolen and Terwilliger, and furthermore, precisely when $\Gamma$ is locally strongly regular with nontrivial eigenvalues $p:=\theta_{2}$ and $-q:=\theta_{3}$. When this is the case, the intersection parameters of $\Gamma$ can be parametrized by $p, q$ and the size of the antipodal classes $r$ of $\Gamma$.

Let $\Gamma$ be an antipodal tight graph of diameter 4 , denoted by $\operatorname{AT4}(\boldsymbol{p}, \boldsymbol{q}, \boldsymbol{r})$, and let the $\boldsymbol{\mu}$-graph be a graph that is induced by the common neighbours of two vertices at distance 2 . Then we show that all the $\mu$-graphs of $\Gamma$ are complete multipartite if and only if $\Gamma$ is $\operatorname{AT} 4(s q, q, q)$ for some natural number $s$. As a consequence, we derive new existence conditions for graphs of the AT4 family whose $\mu$-graphs are not complete multipartite. Another interesting application of our results is also that we were able to show that the $\mu$-graphs of a distance-regular graph with the same intersection array as the Patterson graph are the complete bipartite graph $K_{4,4}$.
\end{abstract}

Keywords Distance-regular graphs · Antipodal · Tight · Locally strongly regular · $\mu$-graphs · AT4 family

\footnotetext{
Authors were supported in part by the $\mathrm{Com}^{2} \mathrm{MaC}-\mathrm{SRC} / \mathrm{ERC}$ program of MOST/KOSEF (grant \# R11-1999-054) and in part by the Slovenian Ministry of Science, while the first author was visiting the Combinatorial and Computational Mathematics Center at POSTECH, and while the second author was visiting the IMFM at the University of Ljubljana.
}

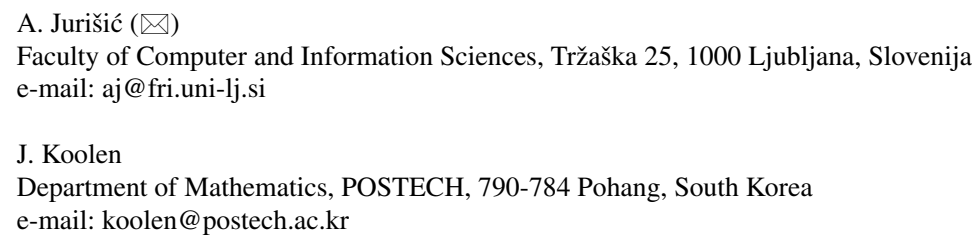




\section{Introduction}

Let $\Gamma$ denote a distance-regular graph with diameter $d \geq 3$, and eigenvalues $k=\theta_{0}>$ $\theta_{1}>\cdots>\theta_{d}$. Jurišić et al. [7,9] showed that the intersection numbers $a_{1}, b_{1}$ satisfy the following inequality

$$
\left(\theta_{1}+\frac{k}{a_{1}+1}\right)\left(\theta_{d}+\frac{k}{a_{1}+1}\right) \geq-\frac{k a_{1} b_{1}}{\left(a_{1}+1\right)^{2}}
$$

and defined $\Gamma$ to be tight whenever it is not bipartite, and equality holds in (1). They also characterized tight graphs in a number of ways, for example by $a_{1} \neq 0, a_{d}=0$ and 1-homogeneous property in the sense of Nomura [14], and furthermore by their first subconstituents being connected strongly regular graphs with nontrivial eigenvalues

$$
b^{+}=-1-\frac{b_{1}}{1+\theta_{d}} \quad \text { and } \quad b^{-}=-1-\frac{b_{1}}{1+\theta_{1}} .
$$

Let $\Gamma$ be a 1-homogeneous graph with diameter $d \geq 2$. Then $\Gamma$ is distance-regular and also locally strongly regular with parameters $\left(v^{\prime}, k^{\prime}, \lambda^{\prime}, \mu^{\prime}\right)$, where $v^{\prime}=k, k^{\prime}=a_{1}$ and $\left(v^{\prime}-k^{\prime}-1\right) \mu^{\prime}=k^{\prime}\left(k^{\prime}-1-\lambda^{\prime}\right)$. Let $\mu$-graph be a graph that is induced by the common neighbours of two vertices at distance 2 . Since a $\mu$-graph of $\Gamma$ is a regular graph with valency $\mu^{\prime}$, (for the local graph of the $\mu$-graph is the $\mu$-graph of the local graph, see [7, Theorem 3(i)]), we have $c_{2} \geq \mu^{\prime}+1$. If $c_{2}=\mu^{\prime}+1$ and $c_{2} \neq 1$, then $\Gamma$ is a Terwilliger graph, i.e., all the $\mu$-graphs of $\Gamma$ are complete. In [10] we classified the Terwilliger 1-homogeneous graphs with $c_{2} \geq 2$ and obtained that there are only three such examples. In [12] we classified the case $c_{2}=\mu^{\prime}+2 \geq 3$, i.e., the case when the $\mu$-graphs of $\Gamma$ are the Cocktail Party graphs, and obtained that either $\lambda^{\prime}=0, \mu^{\prime}=2$ or there are only seven such examples. We show in some less trivial cases that the $\mu$-graphs are complete multipartite, see Table 1 . Our study is part of a larger project to classify 1 -homogeneous graphs whose $\mu$-graphs are complete multipartite.

Table 1 Known examples of the AT4 family, where "!" indicates the uniqueness of the corresponding graph (for the proofs of uniqueness of A4, A6, A8 see [8]). Note $\alpha=(p+q) / r, c_{2}=q \alpha, a_{1}=p(q+1)$, $a_{2}=p q^{2}, n^{\prime}=k, k^{\prime}=a_{1}, \lambda^{\prime}=2 p-q$ and $\mu^{\prime}=p$. For the information on local graphs see [1] and [2]. The local strongly regular graph of A9 has parameters $(416,100,36,20)$ and is the second graph of the Suzuki tower [19], more precisely a rank 3 graph of the group $G_{2}(4): 2$. The local strongly regular graph of A10 has parameters $(31671,3510,693,351)$ and is a rank 3 graph of the sporadic group $F i_{23}$ [2, p. 396]. For the remaining open cases see [6]

\begin{tabular}{llllllllllll}
\hline$\#$ & Graph & $k$ & $p$ & $q$ & $r$ & $\alpha$ & $c_{2}$ & $a_{1}$ & $\lambda^{\prime}$ & $\mu$-Graph & Locally \\
\hline A1 & ! Conway-Smith & 10 & 1 & 2 & 3 & 1 & 2 & 3 & 0 & $K_{2}$ & Petersen \\
A2 & ! J(8,4) & 16 & 2 & 2 & 2 & 2 & 4 & 6 & 2 & $K_{2,2}$ & $K_{4} \times K_{4}$ \\
A3 & ! halved 8-cube & 28 & 4 & 2 & 2 & 3 & 6 & 12 & 6 & $K_{3 \times 2}$ & $T(8)$ \\
A4 & ! 3. $O_{6}^{-}(3)$ & 45 & 3 & 3 & 3 & 2 & 6 & 12 & 3 & $K_{3,3}$ & $G Q(4,2)$ \\
A5 & ! Soicher1 [18] & 56 & 2 & 4 & 3 & 2 & 8 & 10 & 0 & $2 \cdot K_{2,2}$ & Gewirtz \\
A6 & ! 3.O $O_{7}(3)$ & 117 & 9 & 3 & 3 & 4 & 12 & 36 & 15 & $K_{4 \times 3}$ & $N O_{3}^{+}(3)$ \\
A7 & Meixner1 [13] & 176 & 8 & 4 & 2 & 6 & 24 & 40 & 12 & $2 \cdot K_{3 \times 4}$ & $N U(5,2)$ \\
A8 & ! Meixner2 [13] & 176 & 8 & 4 & 4 & 3 & 12 & 40 & 12 & $K_{3 \times 4}$ & $\overline{N U(5,2)}$ \\
A9 & Soicher2 [18] & 416 & 20 & 4 & 3 & 8 & 32 & 100 & 36 & $K_{2}$-ext. of $\frac{1}{2} Q_{5}$ & $G_{2}(4): 2$ \\
A10 & 3.F $i_{24}^{-}[4]$ & 31671 & 351 & 9 & 3 & 120 & 1080 & 3510 & 693 & $O_{8}^{+}(3)$ & $F i_{23}$
\end{tabular}


Let $\Gamma$ be an antipodal distance-regular graph of diameter 4 , with eigenvalues $\theta_{0}>\theta_{1}>\theta_{2}>\theta_{3}>\theta_{4}$. Then its Krein parameter $q_{11}^{4}$ vanishes precisely when $\Gamma$ is tight in the sense of Jurišić, Koolen and Terwilliger, and furthermore, precisely when $\Gamma$ is locally strongly regular with nontrivial eigenvalues $p:=\theta_{2}$ and $-q:=\theta_{3}$. When this is the case, the intersection parameters of $\Gamma$ can be parametrized by $p, q$ and the size of the antipodal classes $r$, so we denote the graph $\Gamma$ by $\mathbf{A T 4}(\boldsymbol{p}, \boldsymbol{q}, \boldsymbol{r})$, see [11] and [7].

Let $\Gamma$ be an $\operatorname{AT} 4(p, q, r)$ graph. We prove that all the $\mu$-graphs of $\Gamma$ are complete multipartite if and only if $\Gamma$ is $\operatorname{AT} 4(s q, q, q)$ for some natural number $s$. As a consequence of the above results we derive new conditions for graphs of the AT4 family whose $\mu$-graphs are not complete multipartite. Another interesting application of our results is also that we were able to show that the $\mu$-graphs of a distance-regular graph with the same intersection array as the Patterson graph are the complete bipartite graph $K_{4,4}$.

Often knowing $\mu$-graphs ends in a complete classification or characterization with intersection array, see for example [2, p. 271, Theorem 9.3.8]. As we will see, the same is true also in the case of the Patterson graph [3] and in the case of the AT4 $(s q, q, q)$ family of distance-regular graphs [8].

\section{Preliminaries}

Let $\Gamma$ be a graph with diameter $d$. For vertices $x_{1}, \ldots, x_{n}$ of $\Gamma$ we denote by $\Gamma\left(x_{1}, \ldots, x_{n}\right)$ the set of their common neighbours and by $\Delta\left(x_{1}, \ldots, x_{n}\right)$ the graph induced by this set. In particular, for a vertex $x$ of $\Gamma$ we call $\Delta(x)$ the local graph of $x$. The graph $\Gamma$ is said to be locally $\mathcal{C}$, where $\mathcal{C}$ is a graph or a class of graphs, when all its local graphs are isomorphic to (respectively are member of) $\mathcal{C}$. For example, the icosahedron is locally a pentagon, and the point graphs of generalized quadrangles are locally a union of cliques.

We define $\Gamma_{i}(x)$ to be the set of vertices at distance $i$ from $x$. For $y \in \Gamma_{i}(x)$ and integers $j$ and $h$ we denote the set $\Gamma_{j}(x) \cap \Gamma_{h}(y)$ by $\boldsymbol{D}_{j}^{\boldsymbol{h}}(\boldsymbol{x}, \boldsymbol{y})$ and its cardinality by $\boldsymbol{p}_{j \boldsymbol{h}}^{i}(\boldsymbol{x}, \boldsymbol{y})$. We say that the intersection number $p_{j h}^{i}$ does exist if $p_{j h}^{i}(x, y)=p_{j h}^{i}$ for all pairs of vertices $x$ and $y$ at distance $i$, i.e., it is independent of a choice of $x$ and $y$ at distance $i$. We denote the intersection numbers $p_{1 i}^{i}, p_{1, i+1}^{i}, p_{1, i-1}^{i}$ and $p_{i i}^{0}$ respectively by $\boldsymbol{a}_{\boldsymbol{i}}, \boldsymbol{b}_{\boldsymbol{i}}, \boldsymbol{c}_{\boldsymbol{i}}$ and $\boldsymbol{k}_{\boldsymbol{i}}$, for $\boldsymbol{i}=0,1, \ldots, d$. The distance-regular graphs are characterized as the graphs for which the set of parameters $\left\{b_{0}, \ldots, b_{d-1} ; c_{1}, \ldots, c_{d}\right\}$, called the intersection array of $\Gamma$, exist, or equivalently when for all $i, j$ and $h$ the numbers $p_{j h}^{i}$ do exist. Note that a distance-regular graph is $k$-regular, where $k=k_{1}=b_{0}$, and $k=a_{i}+b_{i}+c_{i}$. All local graphs have $k$ vertices and are $a_{1}$-regular. More generally, in a distance-regular graph $\Gamma$ for each vertex $x$, the $i$-subconstituent graph of $x$, i.e., the graph induced by the set $\Gamma_{i}(x)$, is $a_{i}$-regular. For a detailed treatment of distance-regular graphs and all the terms which are not defined here see Brouwer et al. [2] or Godsil [5].

Let us now recall that an equitable partition of a graph is a partition $\pi=$ $\left\{P_{1}, \ldots, P_{s}\right\}$ of its vertices into cells, such that for all $i$ and $j$ the number $c_{i j}$ of neighbours, which a vertex in the cell $P_{i}$ has in the cell $P_{j}$, is independent of the choice of the vertex in $P_{i}$. Let $\Gamma$ be a distance-regular graph with diameter $d$. Then $\Gamma$ is 1homogeneous in the sense of Nomura [14], when the distance partition corresponding 
to any pair $x, y$ of adjacent vertices, i.e., the collection of nonempty sets $D_{h}^{j}(x, y)$, is an equitable partition.

Let $\Gamma$ be a graph. As usually, we denote the distance between vertices $x$ and $y$ of $\Gamma$ by $\partial(x, y)$. If $x, y$ and $z$ are vertices of $\Gamma$ such that $\partial(x, y)=1, \partial(x, z)=\partial(y, z)=2$, then we define the (triple) intersection number $\alpha(x, y, z)=|\Gamma(x) \cap \Gamma(y) \cap \Gamma(z)|$ (see Fig. 3(a)). We say that the parameter $\alpha$ of $\Gamma$ exists when $\alpha=\alpha(x, y, z)$ for all triples of vertices $(x, y, z)$ of $\Gamma$ such that $\partial(x, y)=1, \partial(x, z)=\partial(y, z)=2$. If $\Gamma$ is 1-homogeneous graph with diameter $d \geq 2$ and $a_{2} \neq 0$, then $\alpha$ exists. A strongly regular graph with $a_{2} \neq 0$, that is locally strongly regular is 1-homogeneous if and only if $\alpha$ exists. See for example [9, Lemma 2.11].

We end this section with some information on the AT4 family, see [11, 5.2-6.4].

Proposition 2.1. Let $\Gamma$ be an antipodal tight graph $\operatorname{AT} 4(p, q, r)$. Then $q_{11}^{4}=0$ and

(i) $p q+p+q>p>-q>-q^{2}$ are its nontrivial eigenvalues and its intersection array equals

$$
\begin{gathered}
\left\{q(p q+p+q),\left(q^{2}-1\right)(p+1), \frac{(r-1) q(p+q)}{r}, 1 ;\right. \\
\left.1, \frac{q(p+q)}{r},\left(q^{2}-1\right)(p+1), q(p q+p+q)\right\}
\end{gathered}
$$

(ii) the local graphs are connected and strongly regular with eigenvalues $a_{1}, p,-q$ and parameters

$$
\left(k^{\prime}, \lambda^{\prime}, \mu^{\prime}\right)=(p(q+1), 2 p-q, p),
$$

(iii) the graph $\Gamma$ is 1-homogeneous, see Fig. 1 and in particular $\alpha=(p+q) / r$,

(iv) the parameters $p, q, r$ are integers, such that $p \geq 1, q \geq 2, r \geq 2$ and

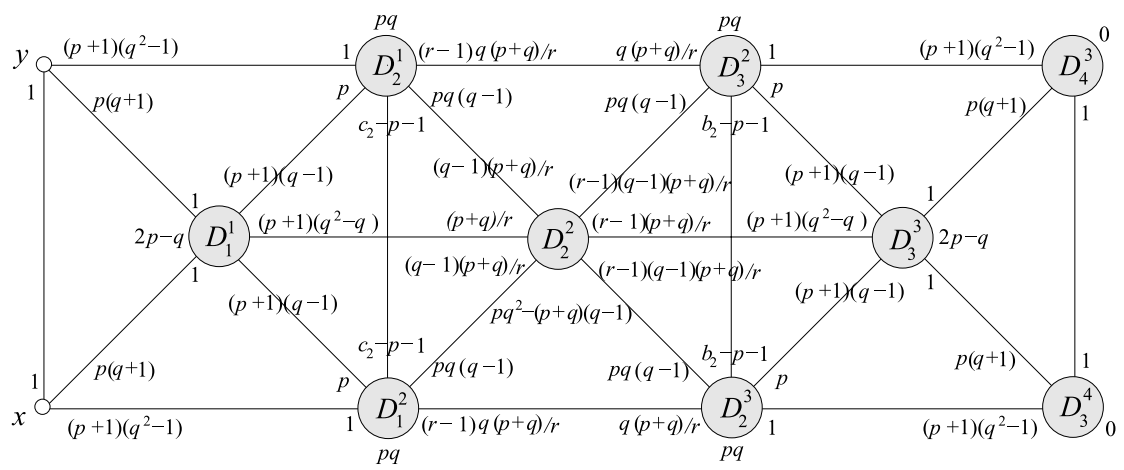

Fig. 1 The distance partition corresponding to an edge $x y$ of $\Gamma$. The number beside edges connecting cells $D_{i}^{j}(x, y)$, indicates how many neighbours a vertex from the closer cell has in the other cell. We also put beside each cell the valency of the graph induced by the vertices of it. For convenience we mention here the intersection numbers needed for the above partition: $\left|D_{1}^{1}\right|=p_{11}^{1}=a_{1}=p(q+1), \quad\left|D_{2}^{1}\right|=$ $p_{12}^{1}=b_{1}=\left(q^{2}-1\right)(p+1), \quad\left|D_{3}^{2}\right|=p_{23}^{1}=(r-1) b_{1}=(r-1)\left(q^{2}-1\right)(p+1), \quad\left|D_{4}^{3}\right|=p_{34}^{1}=r-1$, $\left|D_{2}^{2}\right|=p_{22}^{1}=\operatorname{rpq}\left(q^{2}-1\right)(p+1) /(p+q)$ 
(1) $p q(p+q) / r$ is even, $r(p+1) \leq q(p+q)$, and $r \mid p+q$,

(2) $p \geq q-2$, with equality if and only if $q_{44}^{4}=0$,

(3) $p+q \mid q^{2}\left(q^{2}-1\right)$ and $p+q^{2} \mid q^{2}\left(q^{2}-1\right)\left(q^{2}+q-1\right)(q+2)$.

(v) $(p=) \mu^{\prime}=1$ iff $\alpha=1$ iff $p+q=r$ iff $c_{2}=\mu^{\prime}+1$ iff $r(p+1)=q(p+q)$ iff $\Gamma$ is the unique AT4(1, 2, 3) graph, i.e., the Conway-Smith graph.

\section{Complete multipartite $\mu$-graphs}

There are distance-regular graphs for which it is possible to determine what their $\mu$-graphs are based only on their parameters even when $a_{1} \neq 0$. Let $\Gamma$ be a distance-regular graph with diameter at least 2, for which the parameter $c_{2}$ of its local graphs, denoted by $\mu^{\prime}$, exists. Then the assumption $c_{2}=\mu^{\prime}+1$ is equivalent to all the $\mu$-graphs being complete and the assumption $c_{2}=\mu^{\prime}+2 \geq 3$ is equivalent to all the $\mu$-graphs being Cocktail Party graphs. In both cases the $\mu$-graphs are complete multipartite. We will show in this section that there are more cases where we can assert that the $\mu$-graphs are complete multipartite only based on certain parameter properties.

We start by recalling two definitions and one result [12, Lemmas 2.1 and 3.1] that has already been used for a classification of 1-homogeneous distance-regular graphs with Cocktail Party $\mu$-graphs. We denote the complement of $t$ cliques of size $n$, i.e., the complete multipartite graph $K_{n_{1}, n_{2}, \ldots, n_{t}}$ with $n_{1}=n_{2}=\cdots=n_{t}=n$ by $\boldsymbol{K}_{\boldsymbol{t} \times \boldsymbol{n}}$. If a graph $\Gamma$ on $v$ vertices is regular with valency $k$ and any two vertices of $\Gamma$ at distance 2 have precisely $\mu=\mu(\Gamma)$ common neighbours, then the graph is called co-edge-regular with parameters $(v, k, \mu)$, see [2, p. 3].

Proposition 3.1. Let us fix integers $t$ and $n$, and let $\Gamma$ be a distance-regular graph with diameter at least 2 , whose $\mu$-graphs are the complete multipartite graph $K_{t \times n}$, for which $a_{2} \neq 0$ and the intersection number $\alpha$ exists with $\alpha \geq 1$. Then the following (i)-(iii) hold.

(i) $c_{2}=n t$, for each vertex $x$ of $\Gamma$ the local graph $\Delta(x)$ is co-edge-regular with parameters $\left(v^{\prime}, k^{\prime}, \mu^{\prime}\right)$, where $v^{\prime}=k, k^{\prime}=a_{1}$ and $\mu^{\prime}=n(t-1)$. Moreover, $\alpha a_{2}=c_{2}\left(a_{1}-\mu^{\prime}\right)$.

(ii) Let $x$ and $y$ be vertices of $\Gamma$ at distance 2. Then for all $z \in D_{1}^{2}(x, y) \cup D_{2}^{1}(x, y)$ the subgraph $\Delta(x, y, z)$ is complete and $\alpha \in\{t-1, t\}$.

(iii) Let $\Gamma$ be locally strongly regular with parameters $\left(v^{\prime}, k^{\prime}, \lambda^{\prime}, \mu^{\prime}\right), t \geq 2$ and let $x$ and $z$ be adjacent vertices of $\Gamma$. Then the subgraph $\Delta(x, z)$ is co-edge-regular with parameters $\left(v^{\prime \prime}, k^{\prime \prime}, \mu^{\prime \prime}\right)$, where $v^{\prime \prime}=k^{\prime}, k^{\prime \prime}=\lambda^{\prime}$ and $\mu^{\prime \prime}=n(t-2)$, for $t \geq 3$ the subgraph $\Delta(x, z)$ has diameter 2.

The above result gives also some necessary conditions for a distance-regular graph to have completely regular $\mu$-graphs. Let $\Gamma$ be a distance-regular graph that is locally connected and co-edge-regular. Furthermore, we also assume that the parameter $\alpha$ exists in $\Gamma$. All 1-homogeneous distance-regular graphs with diameter at least 2 have these properties. We provide some sufficient conditions on the parameters of $\Gamma$ for which the $\mu$-graphs have to be complete multipartite. 
Theorem 3.2. Let $\Gamma$ be a distance-regular graph with diameter at least $2, a_{2} \neq 0$, for which the intersection number $\alpha$ exists and that is locally co-edge-regular with parameters $\left(v^{\prime}, k^{\prime}, \mu^{\prime}\right)$, where $v^{\prime}=k, k^{\prime}=a_{1}$ and $c_{2}>\mu^{\prime}+1>1$. Then $\alpha \geq 1$ and the following holds.

(i) If $\alpha=1$ then $c_{2}=2 \mu^{\prime}$ and the $\mu$-graphs are the complete bipartite graphs $K_{\mu^{\prime}, \mu^{\prime}}$.

(ii) If $\alpha>1$ and $2 c_{2}+\alpha<3 \mu^{\prime}+6$, then the $\mu$-graphs are the complete multipartite graph $K_{t \times n}$, where $n=c_{2}-\mu^{\prime}$ and $t=c_{2} / n$.

(iii) If $\alpha=2$ and $c_{2} \leq 2 \mu^{\prime}$, then either $c_{2}=2 \mu^{\prime}$ and the $\mu$-graphs are the complete bipartite graph $K_{\mu^{\prime}, \mu^{\prime}}$ or $c_{2}=3 \mu^{\prime} / 2$ and the $\mu$-graphs are the complete multipartite graph $K_{3 \times \mu^{\prime} / 2}$

Proof: The assumption $\mu^{\prime}>0$ implies that $\Gamma$ is locally connected, thus $\alpha \geq 1$. Let $x$ and $y$ be any two vertices of $\Gamma$ at distance 2. Then the graph $\Delta(x, y)$ has $c_{2}$ vertices, valency $\mu^{\prime}$ by [7, Theorem 3.1(i)], and it is not complete, since we assumed $c_{2}>$ $\mu^{\prime}+1$. Therefore, there are nonadjacent vertices in $\Delta(x, y)$. If any pair of such vertices has $\mu^{\prime}$ common neighbours, then $\Delta(x, y)$ is a complete multipartite graph $K_{t \times n}$, where $n=c_{2}-\mu^{\prime}$ and $t=c_{2} / n$ (cf. [2, p. 3], as it is co-edge-regular and has $k^{\prime}=\mu^{\prime}$ ). Let us now assume that there exist nonadjacent vertices $u$ and $v$ in $\Delta(x, y)$ such that

$$
w:=|\Gamma(x, y, u, v)|<\mu^{\prime}, \quad \text { i.e., } \quad s:=\mu^{\prime}-w=\left|D_{2}^{1}(x, y) \cap \Gamma(u, v)\right| \geq 1,
$$

(see Fig. 2). Let $\Gamma(x, y, u, v)=\left\{z_{1}, \ldots, z_{w}\right\}, \Gamma(u, v) \cap D_{1}^{2}(x, y)=\left\{x_{1}, x_{2}, \ldots, x_{s}\right\}$ and $\Gamma(u, v) \cap D_{2}^{1}(x, y)=\left\{y_{1}, y_{2}, \ldots, y_{s}\right\}$.

(i) Then $\alpha=\left|\Gamma\left(x_{1}, x, y\right)\right| \geq|\{u, v\}|=2$, which is not possible. Hence, the $\mu$-graphs of $\Gamma$ are the complete multipartite graph $K_{t \times n}$. By Proposition 3.1(ii), we have $t=2$ and thus also $c_{2}=2 \mu^{\prime}$.

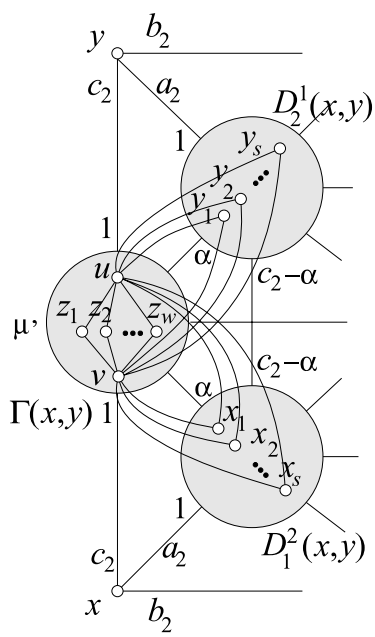

Fig. 2 Part of the distance partition corresponding to vertices $x$ and $y$ at distance 2 
(ii) We have $\Gamma\left(x_{1}, y, u, v\right) \subseteq\left(\Gamma\left(x, x_{1}, y\right) \cap \Gamma(u, v)\right) \cup\left(D_{2}^{1}(x, y) \cap \Gamma(u, v)\right)$, and thus we obtain an upper bound on the size of the set $\Gamma\left(x_{1}, y, u, v\right)$ :

$$
\left|\Gamma\left(x_{1}, y, u, v\right)\right| \leq \alpha-2+s
$$

Note that here we needed the assumption $\alpha \geq 2$. Similarly, we derive two more inequalities:

$$
\begin{aligned}
c_{2}= & |\Gamma(u, v)| \geq\left|\left\{x, y, x_{1}, \ldots, x_{s}, y_{1}, \ldots, y_{s}, z_{1}, \ldots, z_{w}\right\}\right| \\
= & \mu^{\prime}+2+s, \quad \text { i.e., } \quad s \leq c_{2}-\mu^{\prime}-2 \\
& \left|\Gamma\left(y, x_{1}\right)\right| \geq\left|\Gamma\left(x_{1}, y, u\right)\right|+\left|\Gamma\left(x_{1}, y, v\right)\right|-\left|\Gamma\left(x_{1}, y, u, v\right)\right|+|\{u, v\}|, \quad \text { i.e. } \\
& 2 \mu^{\prime}-c_{2}+2 \leq\left|\Gamma\left(x_{1}, y, u, v\right)\right| .
\end{aligned}
$$

Finally, by combining (3) and 5 and assuming (4), we obtain $3 \mu^{\prime}+6 \leq 2 c_{2}+\alpha$, which is contradicting the assumption. So each $\mu$-graph of $\Gamma$ is a complete multipartite graph.

(iii) Since we assumed $c_{2} \leq 2 \mu^{\prime}$, the vertices $u$ and $v$ have a common neighbour in $\Delta(x, y)$. This conclusion translates to $w \geq 1$. Because $\alpha=2$ the only neighbours in $\Gamma(x, y)$ of the vertex $x_{i}$ are $u$ and $v$, so $z_{j}$ is not adjacent to $x_{i}$ or $y_{i}$ for all $i$ and $j$. Therefore,

$$
\begin{aligned}
c_{2} & =|\Gamma(u, v)| \geq\left|\left\{z_{1}, x_{1}, \ldots, x_{s}, y_{1}, \ldots, y_{s}\right\}\right|+\left|\Gamma\left(z_{1}, u, v\right)\right| \\
& =1+2 s+\mu^{\prime} \quad \text { i.e., } \quad c_{2}-1-2 s \geq \mu^{\prime} .
\end{aligned}
$$

In order to get the above inequality we started with two nonadjacent vertices $u$ and $v$ in $\Gamma(x, y)$, where the distance between $x$ and $y$ is 2 and $|\Gamma(u, v, x, y)|=$ $w<\mu^{\prime}$. Now for the nonadjacent vertices $x_{1}$ and $y$ in $\Gamma(u, v)$ we have that the distance between $u$ and $v$ is 2 and $\left|\Gamma\left(x_{1}, y, u, v\right)\right| \leq s$, since $\Gamma(y, u, v)=$ $\left\{y_{1}, y_{2}, \ldots, y_{s}, z_{1}, \ldots, z_{w}\right\}$ and $\Gamma\left(x_{1}, y, u, v\right) \subseteq\left\{y_{1}, y_{2}, \ldots, y_{s}\right\}$. As $s=\mu^{\prime}-$ $w<\mu^{\prime}$ we conclude the same way as in (6) that

$$
c_{2}-1-2 w \geq \mu^{\prime}
$$

However, by summing (6) and (7), and using $\mu^{\prime}=s+w$, we obtain $c_{2}>2 \mu^{\prime}$, a contradiction! Therefore, the $\mu$-graphs of $\Gamma$ are complete multipartite graph $K_{t \times n}$. By $2=\alpha \in\{t-1, t\}$, we have $c_{2}=2 n$ and $\mu^{\prime}=n$ when $t=2$ and $c_{2}=3 n$ and $\mu^{\prime}=2 n$ when $t=3$.

\section{Remark 3.3.}

(i) There are some examples of the graph $\Gamma$ from the above Theorem 3.2 (ii) that are locally co-edge-regular and not locally strongly regular. For example the Johnson graph $J(n, e)$ is locally the grid graph $e \times(n-e)$, which means that it is not locally strongly regular unless $n=2 e$. Let us assume that $e$ and $n-e$ are at least 2. It has $c_{2}=4, \mu^{\prime}=2, a_{1}=n-2$ and $\alpha=2$, so it satisfies Theorem 3.2(ii). 
Hence its $\mu$-graphs are complete bipartite. It would be interesting to find some examples for which $c_{2}>\mu^{\prime}+2$.

(ii) If we want that the $\mu$-graphs of $\Gamma$ are the complete multipartite graph $K_{t \times n}$, then the parameters $n$ and $t$ are determined by $c_{2}=n t$ and $\mu^{\prime}=(t-1) n$, i.e., $n=c_{2}-\mu^{\prime}$ and $t=c_{2} / n$.

Corollary 3.4. Let $\Gamma$ be a 1-homogeneous graph with diameter at least 2 . If $a_{2} \neq 0$, $\alpha=2$ and $c_{2}=2 \mu^{\prime}>2$, then the $\mu$-graphs of $\Gamma$ are the complete bipartite graphs $K_{\mu^{\prime}, \mu^{\prime}}$. In particular, the $\mu$-graphs of a graph with the same intersection array as the Patterson graph are $K_{4,4}$.

Proof: The first part is a straightforward consequence of Theorem 3.2(iii). Let now $\Gamma$ be a distance-regular graph with the intersection array $\{280,243,144,10$; $1,8,90,280\}$, i.e, the one of the Patterson graph. Then its eigenvalues are $280^{1}, 80^{364}, 20^{5940},-8^{15795},-28^{780}$, so it satisfies (1) with equality and is thus tight by [9, Theorems 12.6 and 11.7], it is locally connected, 1-homogeneous, $a_{2}=128$ and $c_{2}=8, \mu^{\prime}=4$ and $\alpha=2$, see [9, Example (xii) and Fig. A.4(k)]. So, by Theorem 3.2(iii), its $\mu$-graphs are the complete bipartite graphs $K_{4,4}$.

Theorem 3.5. Let $\Gamma$ be a distance-regular graph with diameter at least $2, a_{2} \neq 0$, for which the intersection number $\alpha$ exists with $\alpha \geq 1$. Then the following (i) and (ii) are equivalent.

(i) there are such integers $t, n \geq 2$ that the $\mu$-graphs of $\Gamma$ are the complete multipartite graph $K_{t \times n}$,

(ii) there exists a natural number $\mu^{\prime} \geq 1$, such that $\Gamma$ is locally co-edge-regular with parameters $\left(v^{\prime}, k^{\prime}, \mu^{\prime}\right)$, where $v^{\prime}=k, k^{\prime}=a_{1}, c_{2}>\mu^{\prime}+1$ and one of the following (1)-(3) holds:

1. $\alpha=1$,

2. $\alpha=2$ and $c_{2} \leq 2 \mu^{\prime}$,

3. $\alpha \geq 3$ and $2 c_{2}+\alpha<3 \mu^{\prime}+6$.

Suppose (i) and (ii) above hold. Then $n=c_{2}-\mu^{\prime}, t=c_{2} / n$ and $\alpha \in\{t-1, t\}$.

Proof: Let us assume (i) holds. Then, by Proposition 3.1, the graph $\Gamma$ is locally co-edge-regular with parameters $\left(k, a_{1}, \mu^{\prime}\right)$, where $\mu^{\prime}=(t-1) n, c_{2}=n t, \alpha a_{2}=$ $c_{2}\left(a_{1}-\mu^{\prime}\right)$ and $\alpha \in\{t-1, t\}$. If $\alpha=1$, then $t=2, c_{2}=2 \mu^{\prime}, a_{2}=2 n\left(a_{1}-n\right)$ and the $\mu$-graphs of $\Gamma$ are $K_{n, n}$. If $\alpha=2$, then the assumption $t \geq 2$ is equivalent to $\mu^{\prime}>0$, which means that the graph $\Gamma$ is locally connected. It also implies, by $t=c_{2} /\left(c_{2}-\mu^{\prime}\right)$, that we have $c_{2} \leq 2 \mu^{\prime}$. Finally, we assume $\alpha \geq 3$. Then $t \geq 3$ and we have $(n-2)(t-$ $3) \geq 0$, which implies $2\left(c_{2}+\alpha\right) \leq 3 \mu^{\prime}+6$ and so also $2 c_{2}+\alpha<3 \mu^{\prime}+6$.

The rest of the statement follows directly from Theorem 3.2.

Problem 3.6 Find more necessary and sufficient conditions for the graph $\Gamma$ from Theorem 3.2 to have complete multipartite $\mu$-graphs. Or even more generally, find more properties of a distance-regular graph $\Gamma$ that determine its $\mu$-graphs. (There are examples of graphs in the AT4 family that have more complicated $\mu$-graphs, see Table 1.) 


\section{AT4 family}

Let $\Gamma$ be an antipodal tight graph $\operatorname{AT} 4(p, q, r)$. Based on Proposition 2.1, we have $\mu^{\prime}=p>0$ (i.e., $\Gamma$ is locally connected), and $a_{2}=p q^{2} \neq 0$, which means that the following result is a direct consequence of Theorem 3.2 and Proposition 2.1 (the case $p+q=r$ has already been treated in Proposition 2.1(v)).

Corollary 4.1. Let $\Gamma$ be an antipodal tight graph $\mathrm{AT} 4(p, q, r)$ and $p+q>r$. Then the condition

$$
(p+q)(2 q+1)<3 r(p+2)
$$

implies that all $\mu$-graph are the complete multipartite graphs $K_{t \times n}$ with $n=c_{2}-\mu^{\prime}=$ $q \alpha-p$ and $t=c_{2} /\left(c_{2}-\mu^{\prime}\right)=q \alpha /(q \alpha-p)$.

Corollary 4.2. Let $\Gamma$ be an antipodal tight graph $\operatorname{AT} 4(q s, q, q)$, where $s$ is a natural number. Then $\alpha=s+1$ and the $\mu$-graphs are the complete multipartite graphs $K_{(s+1) \times q}$.

Proof: From $p=s q$ and $r=q$ we obtain $\alpha=s+1$ by Proposition 2.1(iii). Let us first assume $s \geq 2$. Then $c_{2}-\mu^{\prime}=\alpha q-s q=q$ and the inequality in Corollary 4.1 translates to $0<(s-2)(q-1)+3$. Since this condition is satisfied, the $\mu$-graphs of $\Gamma$ are the complete multipartite graphs $K_{(s+1) \times q}$.

It remains to consider the case $s=1$. Then $c_{2}=2 q, \mu^{\prime}=q$ and $\alpha=2$. Therefore, by Theorem 3.2(iii), every $\mu$-graph of $\Gamma$ is the complete bipartite graph $K_{q, q}$.

Examples that satisfy the above result are the graphs A2, A3, A4, A6 and A8 from Table 1.1.

Theorem 4.3. Let $\Gamma$ be a tight distance-regular graph $\operatorname{AT} 4(p, q, r)$ with $p>1$. Then its $\mu$-graphs are complete multipartite if and only if there exists an integer s such that $(p, q, r)=(q s, q, q)$.

Proof: Let us assume that $\mu$-graphs of $\Gamma$ are complete multipartite graphs $K_{t \times n}(t$ and $n$ are determined as we know the size of the $\mu$-graph and its valency). Thus, by Proposition 2.1(i-iv) and Proposition 3.1(i-ii), we have $\alpha=(p+q) / r, \alpha \in\{t-1, t\}$,

$$
q \alpha=c_{2}=n t, \quad \text { and } \quad p=\mu^{\prime}=(t-1) n .
$$

Case $\alpha=t$. Then we have $n=q$ and thus also $p=(t-1) q$, i.e., $p+q=t q$. But then $t r=\alpha r=p+q$ implies $r=q$. Hence $(p, q, r)=(s q, q, q)$ for $s=t-1$.

Case $\alpha=t-1$. Then, by (8), we have $q \alpha=n(\alpha+1)$ and $p=\alpha n$. Therefore,

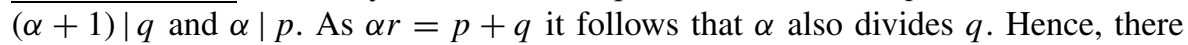
exists a natural number $h$ such that $q=h(\alpha+1) \alpha$. It follows $n=h \alpha^{2}, p=h \alpha^{3}$ and $r=(p+q) / \alpha=h\left(\alpha^{2}+\alpha+1\right)$. Since we assumed $p \neq 1$, we have $\alpha \neq 1$ by Proposition 2.1(v). If $\alpha=2$, then $q=6 h, p=8 h$ and, by Proposition 2.1(iv)(3), we 

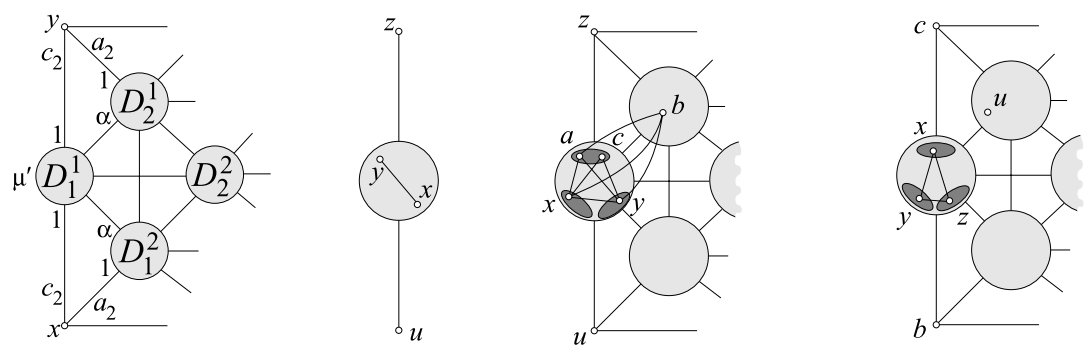

Fig. 3 Parts of the distance distribution diagram of a pair of vertices at distance 2. For the complete figure in the case of diameter 4 see [6, Fig. 6.1]

have $7 \mid(h-1) h(h+1)$, i.e., $h=7 \ell-1$ or $h=7 \ell$ or $h=7 \ell+1$ for an integer $\ell$, and hence $9 \ell-1 \mid 20$ or $2+63 \ell \mid 140$ or $11+63 \ell \mid 140$, which is impossible.

Finally, we assume $\alpha \geq 3$. In this case we first show the following inequality

$$
\lambda^{\prime} \geq 1+(t-2) n+(n-1)((t-3) n-(\alpha-3)) .
$$

Let $x, y$ and $z$ be pairwise adjacent vertices of $\Gamma$. The local graph $\Delta(y)$ is connected and strongly regular with $\lambda^{\prime}=2 p-q=h \alpha(\alpha-1)(2 \alpha+1)$ by Proposition 2.1(ii). Therefore, there exists a vertex $u \in U:=\Gamma(x, y) \cap \Gamma_{2}(z)$, see Fig. 3(b).

Vertices of the graph $\Delta(x, y, z)$ are partitioned into the following sets:

$$
A:=\Gamma(x, y, z, u) \quad \text { and } \quad B:=\Gamma_{2}(u) \cap \Gamma(x, y, z)
$$

so we have $|A|=c_{2}-2 n=(t-2) n$ and $|B|=\lambda^{\prime}-(t-2) n=h \alpha\left(\alpha^{2}-1\right) \neq 0$. Let $b \in B$. By Proposition 3.1(ii), the vertex $b$ has exactly $\alpha-2$ neighbours in $A$, see Fig. 3(c). Since $\alpha \geq 3$, there exists an element $a \in A$ adjacent to $b$. Let $C$ be a maximal independent set in $A$ containing $a$. Let $c \in C \backslash\{a\}$. Note that there are $n-1>0$ choices for $c$. By Proposition 3.1(ii), vertices $a$ and $c$ have no common neighbours in $B$, so the distance between vertices $b$ and $c$ is 2, see Fig. 3(d). Since the $\mu$-graph of $b$ and $c$ is $K_{t \times n}$ and it contains $x, y$ and $z$, the number of common neighbours of $b$ and $c$ in $\Delta(x, y, z)$ is exactly $(t-3) n$. By Proposition 3.1(ii) and $u \in D_{1}^{2}(c, b)$, exactly $\alpha-3$ of those neighbours are adjacent to $u$, hence $|B \cap \Gamma(b, c)|=(t-3) n-(\alpha-3)$. As we have already mentioned, the vertices in $C \backslash\{a\}$ have no common neighbours in $B$, so it follows that $b$ has at least $(n-1)((t-3) n-(\alpha-3))$ neighbours in $B$. As the size of the set $B$ is $\lambda^{\prime}-(t-2) n$, the inequality (9) follows. However, (9) is equivalent to

$$
(3-\alpha)\left(\alpha^{4} h^{2}+\alpha^{3} h^{2}+1\right)-(5 \alpha+1) \alpha h-3 \alpha^{3} h(h-1)-1 \geq 0,
$$

which is clearly impossible. The converse follows directly from Corollary 4.2. 
We have seen in the proof of Theorem 4.3 that the case $\alpha=t-1$ was ruled out. Furthermore, by Theorem 3.2(iii), $\alpha=2$ implies $t=2$, so in the case of $t=3$ we have $\alpha=3$. We propose the following open problem.

Problem 4.4 Let $\Gamma$ be a distance-regular graph with diameter at least 2, whose $\mu$ graphs are the complete multipartite graph $K_{t \times n}$, with $n \geq 2$, for which $a_{2} \neq 0$ and the intersection number $\alpha$ exists with $\alpha \geq 2$. Then show $\alpha=t$.

Corollary 4.5. Let $\Gamma$ be an antipodal tight graph $\operatorname{AT} 4(p, q, r)$. Then exactly one of the following statements holds.

(i) $\Gamma$ is the unique AT4(1, 2, 3) graph (and $\alpha=1)$, i.e., the Conway-Smith graph.

(ii) $\Gamma$ is an $\operatorname{AT4}(q-2, q, q-1)$ graph (and $\alpha=2$ ).

(iii) $\Gamma$ is an $\operatorname{AT} 4(q s, q, q)$ graph, where $s$ is an integer (and $\alpha=s+1)$.

(iv) $(p+q)(2 q+1) \geq 3 r(p+2)$ and $\alpha \geq 3$, in particular $r \leq q-1$.

Proof: If $\alpha=1$, then, by Proposition 2.1(v), the graph $\Gamma$ is the Conway-Smith graph, $p=1, q=2$ and $r=q+1=3$. If $r=q$, then $q$ divides $p$ by Proposition 2.1(iii), and the graph $\Gamma$ is a member of the family $\operatorname{AT} 4(q s, q, q)$ with $\alpha=s+1$ for an integer $s$ by Corollary 4.2 .

Suppose from now on $\alpha \geq 2$ and the graph $\Gamma$ is not a member of the family mentioned in (iii), i.e., the $\mu$-graphs of $\Gamma$ are not all complete multipartite by Theorem 4.3 and Proposition 2.1(v). Let us assume first $\alpha=2$, i.e., $2 r=p+q$ by Proposition 2.1(iii). Then $2 \mid(p+q)$ and we have $2 q=c_{2}>2 \mu^{\prime}=2 p$, i.e., $p+1 \leq q$, by Proposition 2.1 and Theorem 3.2(iii). By Proposition 2.1(iv(2)), this implies $q=p+2$ and $r=q-1$, so the graph $\Gamma$ is a member of the family $\operatorname{AT} 4(q-2, q, q-1)$. Now we assume $3 \leq \alpha$, and we obtain the first inequality in (iv) by Corollary 4.1. Suppose $r>q-1$, i.e., $r \geq q$. Since $r \neq q$, we have $r \geq q+1$. By $\alpha \geq 3$ we obtain $p \geq 2 q+3$. On the other hand, by the first inequality in (iv), we have $2 q^{2} \geq p q+2 p+5 q+6$, i.e., $2 q \geq p+5+2(p+3) / q$, which is not possible.

\section{Remark 4.6.}

(i) The parameters of the family AT4 $\left(h \alpha^{3}, h \alpha(\alpha+1), h\left(\alpha^{2}+\alpha+1\right)\right)$ satisfy the inequality $2 c_{2}+\alpha<3 \mu^{\prime}+6$, hence, by Corollary 4.1 , its members (if they existed) would have complete multipartite $\mu$-graphs. There are some members of this family that passed all other known criteria of feasibility and for which the above statement shows that the corresponding graph does not exist. For example for $h=\alpha(\alpha+1)$ and $(\alpha+2) \mid 570$ we obtain 13 feasible parameter sets, the smallest one being AT4(324, 144, 156), where $\alpha=3$.

(ii) Let us assume $\alpha \geq 3$. Then the first inequality in Corollary 4.5(iv) implies in the case when $p / q$ is large that we can determine all feasible parameter sets using Proposition 2.1(iv(3)). In particular, let us suppose $r=q-1$. By $\alpha=(p+q) / r$, i.e., $p+1=(\alpha-1)(q-1)$, and Corollary 4.5(iv), we obtain

$$
3 \leq \alpha \leq 3+\frac{6}{q-4} .
$$


For $q>10$ we have $6 /(q-4)<1$ and thus $\alpha=3$ and $p=2 q-3$. But, by Proposition 2.1(vi(3)), we have $(q+3) \mid 90$, i.e., $q \in\{12,15,27,42\}$. It is not difficult to consider also the cases when $q \leq 10$.

(iii) There are many parameter sets with $r=q+1, q \neq 2$, that passed all other known criteria and for which the above statement shows that the corresponding graph does not exist. For example, in the case $\alpha=6$ we have AT4(41, 7, 8), AT4(66, 12, 13), AT4(191, 37, 38), AT4(216, 42, 43), and AT4(30s + 21, $6 s+3,6 s+4)$, where $s+1$ is a positive divisor of 60 , i.e., $s \in\{0,1,2,3,4,5,9,11,14,19,29,59\}$. But there are also examples with different $\alpha$ : AT4(519, 36, 37), AT4(1162, 42, 43), AT4(2591, 73, 74).

(iv) The graph A5 is a member of the family $\operatorname{AT} 4(q-2, q, q-1)$, while the graph A9 is an example of AT4( $q s, q, q-1)$, so it satisfies the bound $r \leq q-1$ with equality.

(v) For $\alpha=3$ there exists a feasible family AT4 $(6 s, 6 s, 4 s)$ with $s$ integral, the smallest example is AT4 $(6,6,4)$ with $k=288$ and $a_{1}=42$, cf. B9 and B10 from $[6$, Table 2(b)].

We will give a complete classification of the $\operatorname{AT} 4(q s, q, q)$ family of distanceregular graphs in a subsequent paper [8].

Acknowledgment We would like to thank Leonard Soicher for his construction of the Meixner graphs [16] using GAP [15] and GRAPE [17].

\section{References}

1. A.E. Brouwer, "Strongly regular graphs," in: The CRC Handbook of Combinatorial Designs, (C.J. Colbourn and J.H. Dinitz (Eds.), CRC Press, 1996, pp. 667-685.

2. A.E. Brouwer, A.M. Cohen, and A. Neumaier, Distance-Regular Graphs, Springer-Verlag, Berlin, Heidelberg, Berlin, Heidelberg, (1989) (for corrections and additions see http://www.win.tue.nl/math/ dw/personalpages/aeb/drg/index.html

3. A.E. Brouwer, A. Jurišić and J.H. Koolen, Characterization of the Patterson graph, in preparation.

4. J.T.M. van Bon and R. Weiss, "An existence lemma for groups generated by 3-transpositions," Invent. Math. 109, (1992) 519-534.

5. C.D. Godsil, Algebraic Combinatorics, Chapman and Hall, New York, 1993.

6. A. Jurišić, "AT4 family and 2-homogeneous graphs," Discrete Math. 264(1-3) (2003), 127-148.

7. A. Jurišić and J. Koolen, "Nonexistence of some antipodal distance-regular graphs of diameter four," Europ. J. Combin. 21 (2000), 1039-1046.

8. A. Jurišić and J. Koolen, "Classification of the $\operatorname{AT} 4(q s, q, q)$ family of distance-regular graphs," in preparation.

9. A. Jurišić, J. Koolen, and P. Terwilliger, "Tight distance-regular graphs,” J. Alg. Combin. 12 (2000), $163-197$.

10. A. Jurišić and J. Koolen, "A local approach to 1-homogeneous graphs," Designs, Codes and Cryptography 21 (2000), 127-147.

11. A. Jurišić and J. Koolen, "Krein parameters and antipodal tight graphs with diameter 3 and 4," Discrete Math. 244 (2002), 181-202.

12. A. Jurišić and J. Koolen, "1-homogeneous graphs with Cocktail Party $\mu$-graphs," J. Alg. Combin. 18 (2003), 79-98.

13. T. Meixner, "Some polar towers," Europ. J. Combin. 12 (1991), 397-415.

14. K. Nomura, "Homogeneous graphs and regular near polygons," J. Combin. Theory Ser. B 60 (1994), 63-71. 
15. M. Schönert et al., GAP-Groups, Algorithms and Programming, 4th edition, Lehrstuhl D für Mathematik, RWTH Aachen, 1994.

16. S. Rees and L.H. Soicher, "An algorithmic approach to fundamental groups and covers of combinatorial cell complexes," Symbolic Computation 29 (2000), 59-77.

17. L.H. Soicher, "GRAPE: A system for computing with graphs and groups," in Groups and Computation, L. Finkelstein and W.M. Kantor (Eds.), DIMACS Series in Discrete Mathematics and Theoretical Computer Science vol. 11, A.M.S. 1993 pp. 287-291.

18. L.H. Soicher, "Three new distance-regular graphs," Europ. J. Combin. 14 (1993), 501-505.

19. M. Suzuki, “A finite simple group of order 448,345,497,600" in Symposium on Finite Groups, R. Bauer and C. Sah (Eds), Benjamin, New York, 1969, pp. 113-119. 